\title{
Three-Dimensional Fluorescent Security Features Fabricated via 3D Laser Lithography
}

\author{
Frederik Mayer, Stefan Richter, Phillip Hübner, Toufic Jabbour, \\ and Martin Wegener
}

\begin{abstract}
In the field of optical security features, which are commonly used to protect products or documents against forgery, there is an ongoing race between researchers developing new security features and counterfeiters finding new methods to fake them. Thus, security features continuously need to be improved.
\end{abstract}

Today's optical security features are usually based on planar (2D) fabrication techniques. To further improve the level of counterfeiting security, we have recently proposed, fabricated and characterized three-dimensional fluorescent security features, which are 3D-printed by Direct Laser Writing [1]. These security features consist of a non-fluorescent support structure, into which different fluorescent markers containing $\mathrm{CdSSe} / \mathrm{ZnS}$ quantum dots with different emission wavelengths can be printed in any arbitrary pattern. Thus, data can be stored in the security feature. The structures can be read out by optical sectioning methods. We use confocal laser scanning microscopy, and show fluorescence images as well as well as 3D-reconstructions calculated from the whole fluorescence image stack.

\section{Reference}

1. Mayer F, Richter S, Hübner P, Jabbour T, Wegener M (2017) 3D fluorescence-based security features by 3D laser lithography. Adv Mater Technol 2(11):1700212

\footnotetext{
F. Mayer $(\bowtie) \cdot M$. Wegener Institute of Nanotechnology (INT), Karlsruhe Institute of Technology (KIT), Karlsruhe, Germany Institute of Applied Physics (APH), Karlsruhe Institute of Technology (KIT), Karlsruhe, Germany e-mail: frederik.mayer@kit.edu

S. Richter · P. Hübner

Carl Zeiss AG, Jena, Germany

T. Jabbour

Carl Zeiss AG, Oberkochen, Germany
}

B. Di Bartolo et al. (eds.), Quantum Nano-Photonics, NATO Science for Peace and Security Series B: Physics and Biophysics, https://doi.org/10.1007/978-94-024-1544-5_39 